effusion (serous otitis media, or glue ear) is not clearly made. That commercial pressure is considerable is evidenced by the fact that in a recent large American study the New England Fournal of Medicine received two articles based on the same subjects but submitted by two different groups of investigators. ${ }^{5}$ Both groups had participated in designing and carrying out the study but they could not agree on the results. One group thought that the data statistically supported the use of antibiotics whereas the other group did not. The article supporting antibiotic treatment was the one that was published.

Most likely the answer is that in most children antibiotics make no difference to the outcome in either the short or the long term. More than $85 \%$ of the children will be pain free within 24 hours irrespective of whether they have been treated with an antibiotic or not. ${ }^{2}$ Such treatment is not without side effects - for example, some children develop diarrhoea. The evidence that antibiotics reduce the incidence of mastoiditis is negligible, and indeed this would be hard to prove as the current risk of this occurring when no antibiotics are prescribed is around $0.04 \% .{ }^{26}$ Equally, though the impression is that chronic otitis media is less common than it used to be, there is no evidence to support this once socioeconomic factors have been controlled for (S Gatehouse, proceedings of the eighth international congress in audiology, Jerusalem, 1988).

Thankfully, though often claimed, there is also no evidence that antibiotic treatment increases the risk of subsequent otitis media with effusion even if the course is incomplete. This occurs in $10 \%$ of children after acute otitis media irrespective of treatment. ${ }^{2}$

Finally, in Scandinavian countries antibiotics are not commonly given for childhood otalgia and therefore parents seem not to expect their child to be prescribed them. It is thus a matter of education. If doctors could only more confidently state that antibiotics are unneces-

sary in most instances then parents would normally accept this without question.

\title{
Alternative management strategy
}

So far I have been fairly critical of what often happens based on anecdote. What alternative is suggested based on science? Whether the cause of acute otalgia in childhood is diagnosed correctly is perhaps irrelevant. By definition, all children will be in pain so an analgesic such as a paracetamol elixir will be necessary, irrespective of the cause. In addition, a mild antihistamine sedative, perhaps with a systemic decongestant, will at least allow everyone including the parents to get to sleep, even though it might have no effect on the condition. ${ }^{78}$ In $85 \%$ of children with acute otitis media the otalgia will have subsided within 24 hours irrespective of whether they have received an antibiotic or not. ${ }^{2}$ Antibiotic treatment should be considered only in those who still have otalgia after 24 hours. If this policy were to be generally followed in the United Kingdom 1.84 million fewer prescriptions for antibiotics would be given for childhood otalgia ${ }^{9}$ at a saving of around £6m a year (Viewdate Drug Information Systems, Edinburgh Royal Infirmary, unpublished data).

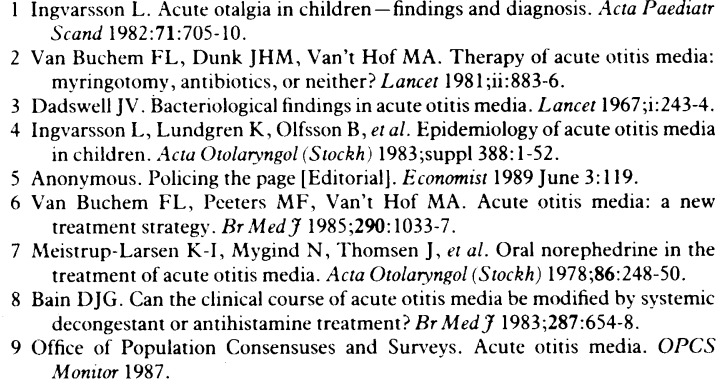

\section{Presentation}

In childhood acute earache is most commonly due to infection of the middle ear, otherwise known as acute otitis media. One in four children will have an episode of acute otitis media at some time during the first 10 years of life, the peak incidence occurring between the ages of 3 and 6 years. ${ }^{-1}$

The onset of earache is often rapid and distressing, with advice from the general practitioner often being sought urgently. In infants and toddlers the combination of pain with associated upper respiratory symptoms causes stress for parents who are naturally unsure of how best to comfort a screaming and fractious child General practitioners are often faced with children during the early stages of the disease when precise diagnostic accuracy is, and has to be, less exacting than that outlined by textbooks.

Examining the tympanic membrane in children is difficult, with limited opportunities to achieve a full view of the eardrum. A fleeting glimpse of the drum may show a leash of prominent blood vessels along the handle of the malleus (see figs), and any hint of inflammation is sufficient to encourage many doctors to diagnose an underlying bacterial infection. Are "painful red eardrums" always due to underlying bacterial infection? There is little consensus about which appearances are diagnostic, but a bulging eardrum has frequently been cited as one of the most useful indicators. ${ }^{67}$ Any sign of inflammation of the eardrum is likely, however, to lead most general practitioners to make a presumptive diagnosis of acute otitis media.

\section{To treat or not to treat?}

With no hard and fast rules about which combination of symptoms and signs are associated with the presence of organisms such as Streptococcus pneumoniae and Haemophilus influenzae, treatment poses several dilemmas. For general practitioners the fleeting hour is a constant enemy and explaining to parents the use of analgesics along with a "wait and see" policy is often more difficult than reaching for the prescription pad and issuing a combination of antibiotic and decongestant.

The standard approach to a rapid onset of earache is a seven to 10 day course of a broad spectrum antibiotic, but only half of all children will complete a one week course. Recent studies have shown that short courses (two to three days of antibiotic) at conventional or high doses are equally effective in terms of resolution of symptoms and signs. ${ }^{910}$

Studies from Scandinavia and The Netherlands have shown that antibiotics are not essential in the treatmen of all infections of the middle ear. ${ }^{112}$ The problem for general practitioners is teasing out which children are 


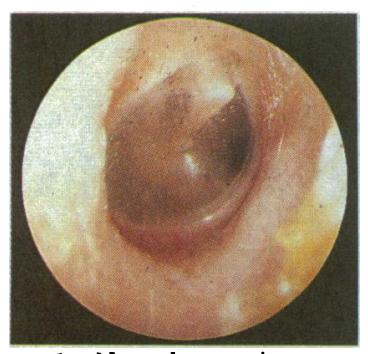

FIG 1-Normal tympanic membrane

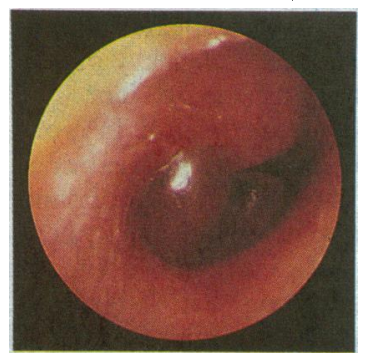

FIG 2-Bulging red eardrum:

antibiotic indicated

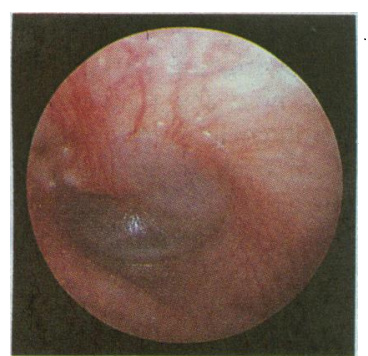

FIG 3-Leash of blood vessels on eardrum: use of antibiotics doubtful likely to develop recurrent problems and in which children antibiotic treatment will reduce recurrences of middle ear infection.

There is little evidence to show that decongestant and antihistamine mixtures relieve the associated symptoms of acute otitis media or improve eustachian tube dysfunction. ${ }^{1314}$ In addition, side effects such as irritability, nightmares, and visual hallucinations have been reported. ${ }^{15}{ }^{16}$ Despite this, many ear, nose, and throat surgeons still advocate their use.

Follow up at six to eight weeks after an acute episode is advisable in young children. When attacks have recurred subsequent review depends on individual circumstances but has to include an assessment of hearing as well as examination of the eardrum. Most children with episodic middle ear infections will not develop serious problems, and the trend in some quarters towards increasing referrals for specialist opinion has to be questioned. A spiral of referral followed by unproved surgical intervention is not always in the best interests of many children with self limiting problems. ${ }^{17}$

\section{Clinical judgment}

The term acute otitis media is often a label to justify the use of antibiotics rather than a true reflection of the underlying disease. This is understandable given the nature of primary medical care, where it is false to assume that general practitioners have access to full knowledge of probabilities, events, and outcomes in caring for the wide range of undifferentiated problems presented to them. Merely condemning general practitioners for apparent overprescribing is insufficient without an understanding of the close interplay of physical, psychological, and social features of children's presenting problems. Before taking decisions on the use of drugs parental expectations have to be explored with the reminder that "diseases fall into new and more relevant groups when illness is seen in its family context."

Research on disorders of the middle ear has been largely confined to hospitals, and a better understanding of the natural course of otitis media can be achieved only by conducting more of the original work in general practice. The traditional model of biomedical research does not always fit easily into the primary care setting, where critical inquiry has to combine investigation of presenting symptoms and signs in conjunction with analysis of decision making by general practitioners. This research has also to include methods to promote improvements in the delivery of primary care by general practitioners, health visitors, and practice nurses. Shared policies in group practice are the exception rather than the norm. It is not a question of what is right or wrong but more a matter of ensuring that the reasons for decisions on management are both understood and thought through.

The photographs are reproduced by permission of MTP Press, Lancaster, from Colour Atlas of Mouth, Throat and Ear Disorders by J Bain, P Carter, and R Morton (1985).

1 Medical Research Council. Acute otitis media in general practice. Lancet 1957;ii:510-4.

2 Lowe JF, Bamforth JS, Pracy R. Acute otitis media: one year in general practice. Lancet $;$ ii: 1129-32.

3 Howie VM, Ploussard JG, Sloyer J. The otitis prone condition. Am f Dis Child 1975;129:676.

4 Paradise JL. Otitis media in infants and children. Pediatrics 1980;65:917-43. 5 Fry J. Common diseases: their nature, incidence and care. 3rd ed. Lancaster: MTP Press, 1983.

6 Halsted C, Lepon ML, Balassanian N, et al. Otitis media: clinical observations, microbiology and evaluation of therapy. Am $\mathcal{J}$ Dis Child 1968;115: $542-51$.

7 Bluestone $\mathrm{CD}$. Recent advances in the pathogenesis, diagnosis and management of otitis media. Pediatr Clin North Am 1981;28:727-55.

8 Pitts J. Shared decision making in the informed treatment of acute otitis media. Practitioner 1987;231:1232-3.

9 Bain DJG, Murphy E, Ross F. Acute otitis media: clinical course among children who received a short course of high dose antibiotic. $\mathrm{Br} \mathrm{Med} \mathcal{F}$ 1985;291:1243-6.

10 Jones RH, Bain DJG. Three day and seven day treatment in acute otitis media: a double blind antibiotic trial. $\mathcal{F} R$ Coll Gen Pract 1986;36:356-8.

11 Van Buchem FL, Peeters MF Van't Hof MA. Acute otitis media: a new treatment strategy. Br Med F 1985;290:1033-7.

12 Mygind N, Meistrup-Larsen KI, Thomsen J, et al. Penicillin in acute otitis media: a double-blind placebo controlled trial. Clin Otolaryngol 1981:6:5-13.

3 Bain DJG. Can the clinical course of acute otitis media be modi decongestant or antihistamine treatment? Br Med $\mathcal{F}$ 1983;287:654-6.

14 Cantekin EI, Mandell EM, Bluestone CD, et al. Lack of efficacy of a decongestant-antihistamine combination for otitis media in children. $N$ Engl decongestant-antihistaminec

15 Lewith GT, Davidson F. Dystonic reactions to Dimotapp elixir. $f R$ Coll Gen Pract 1981;31:241.

16 Sankey RJ, Nunn AJ, Sills JA. Visual hallucinations in children receiving decongestants. Br Med F 1984;288:1369.

7 Black NA. Glue ear: the new dyslexia. BrMed $\mathcal{F}$ 1985;290:1963-5.

18 Miller FJW, Court SDM, Walton WS, Knox EG. Growing up in Newcastle upon Tyne 1960. Oxford: Oxford University Press.

\section{ANY QUESTIONS}

\section{What is the most appropriate site for taking arterial blood for gas sampling?}

In most cases blood samples from anywhere in the systemic arterial tree will yield comparable results on blood gas analysis. The choice of the site for sampling is therefore governed by other factors, not least of which are the skill and experience of the doctor concerned. When sampling with a needle it is logical to use an artery that is superficial, easily palpated (and easily compressed after puncture), and relatively immobile. A paucity of veins in the vicinity reduces the likelihood of misleading results from venous sampling, and the absence of any large nerves reduces the possibility of nerve injury. It is also wise to select an artery with a good collateral flow to minimise the small risk of ischaemic complications.

The site which most closely meets these requirements is the radial artery at the wrist. It is preferable to select the non-dominant hand, particularly in patients who will be using the arm soon afterwards. Only in the rare circumstances of pulmonary hypertension with right to left shunting through a patent ductus arteriosus may the composition of radial arterial blood differ between the two sides. This discrepancy occurs if a proportion of the shunted, unsaturated blood enters the left subclavian artery. This is likely to be encountered only in children, particularly in the neonatal period while the circulation remains transitional. Under such circumstances blood sampled anywhere beyond the aortic arch will always differ from that taken from the right radial artery. The latter, being preductal, will reflect the composition of blood perfusing the head and neck, while a postductal sample will reflect that of blood perfusing the rest of the body.
Puncture of the radial artery may produce pain for some hours afterwards even if local anaesthetic and a small needle are used. ${ }^{1}$ It has been suggested anecdotally that puncture of the brachial artery may be less painful, though there is no good evidence for this. While an Allen test is frequently performed to confirm an adequate ulnar collateral flow, the result does not necessarily predict ischaemic damage. ${ }^{2}$ If for any reason radial arterial sampling is impracticable the brachial, femoral, or dorsalis pedis arteries may be used.

If frequent repeated samples are necessary or if arterial pressure requires constant monitoring an indwelling cannula may be indicated. In general, the same considerations will apply when selecting a suitable site for cannulation, though in some circumstances there may be reasons for choosing a larger artery. ${ }^{3}-\mathrm{M}$ S NIELSEN, consultant anaesthetist, Southampton

1 Clark GS, Latto IP, Davies JM. Symptoms following radial artery puncture. Anaesthesia 1982;37:78-9.

2 Wilkins RG. Radial artery cannulation and ischaemic damage: a review. Anaesthesia 1985;40: 896-9.

Gurman GM, Kriemerman S. Cannulation of big arteries in critically ill patients. Crit Care Med $1985 ; 13: 217-20$

\section{Correction}

Ischaemic heart disease

In the Any Question answered by Dr G R. Thompson about an 8 year old child with a family history of ischaemic heart disease ( 24 March, $p 806$ ) the penultimate sentence should read: "I would not recommend using a hydroxy methyl glutaryl coenzyme A reductase inhibitor in a young girl but this might be considered in later life." We apologise for this editorial error. 